\title{
Self-Switching Kerr Oscillations of Counterpropagating Light in Microresonators
}

\author{
Michael T. M. Woodley $\odot,{ }^{1,2,3,{ }^{*}}$ Lewis Hill ${ }^{1,4}$ Leonardo Del Bino ${ }^{1,2,5}$ Gian-Luca Oppo ${ }^{4},{ }^{4}$ and Pascal Del'Haye $\odot^{5,6, \dagger}$ \\ ${ }^{1}$ National Physical Laboratory, Hampton Road, Teddington TW11 OLW, United Kingdom \\ ${ }^{2}$ SUPA and Department of Physics, Heriot-Watt University, Edinburgh EH14 4AS, United Kingdom \\ ${ }^{3}$ Department of Physics, Blackett Laboratory, Imperial College London, London SW7 2AZ, United Kingdom \\ ${ }^{4}$ SUPA and Department of Physics, University of Strathclyde, 107 Rottenrow, Glasgow G4 ONG, United Kingdom \\ ${ }^{5}$ Max Planck Institute for the Science of Light, Staudtstr. 2, 91058 Erlangen, Germany \\ ${ }^{6}$ Department of Physics, Friedrich Alexander University Erlangen-Nuremberg, 91058 Erlangen, Germany
}

(Received 5 May 2020; revised 16 October 2020; accepted 28 December 2020; published 29 January 2021)

\begin{abstract}
We report the experimental and numerical observation of oscillatory antiphase switching between counterpropagating light beams in Kerr ring microresonators, where dominance between the intensities of the two beams is periodically or chaotically exchanged. Self-switching occurs in balanced regimes of operation and is well captured by a simple coupled dynamical system featuring only the self- and crossphase Kerr nonlinearities. Switching phenomena are due to temporal instabilities of symmetry-broken states combined with attractor merging, which restores the broken symmetry on average. Self-switching of counterpropagating light is robust for realizing controllable, all-optical generation of waveforms, signal encoding, and chaotic cryptography.
\end{abstract}

DOI: 10.1103/PhysRevLett.126.043901

In 1981, Kaplan and Meystre theoretically studied Kerr ring resonators pumped with two input optical beams, of equal intensity and frequency, which enter the resonator in opposing directions [1]—see Fig. 1(a). Resonant build-up then occurs, and the intracavity fields interact through Kerr cross-phase modulation. A key phenomenon that can occur in this bidirectionally pumped system above a certain threshold is a spontaneous symmetry breaking of the circulating field intensities-i.e., the sudden change from equal intensities (a state that becomes unstable) to a situation in which the intensity of one field becomes dominant while the other is suppressed [1-10]. Microresonators can additionally exhibit oscillatory behaviors due to a variety of mechanisms, such as thermal instabilities [11] or external forcing [12]. Here we present the first experimental observation of oscillatory antiphase switching between counter-propagating light beams in a passive Kerr resonator-whereby the intensities of the two fields exchange dominance. In photonics, noisy [13] and chaotic [14] switching between two polarization states, as well as in-phase and antiphase frequency combs [15], have been described in semiconductor lasers. Recently, similar effects have also been described in the simulation of drivendissipative dimers of Bose-Einstein condensates [16].

Published by the American Physical Society under the terms of the Creative Commons Attribution 4.0 International license. Further distribution of this work must maintain attribution to the author(s) and the published article's title, journal citation, and DOI. Open access publication funded by the Max Planck Society.
By scanning the frequency of the input laser in our passive microresonator, spontaneous symmetry breaking between the two counterpropagating beams (leading to a dominant and a suppressed mode) is followed by the onset

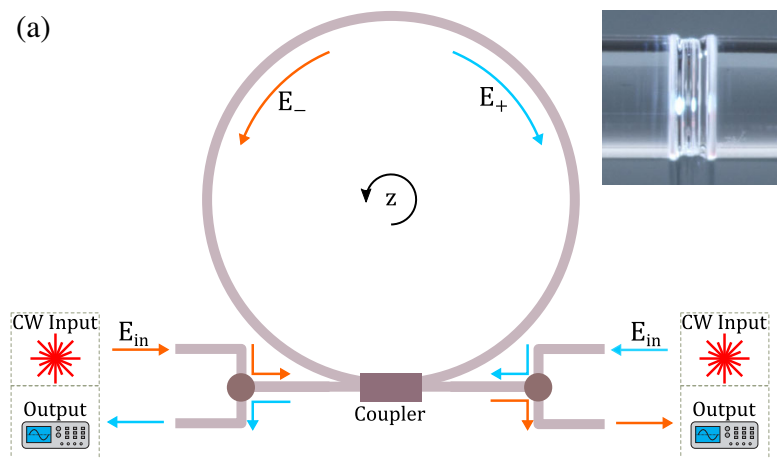

(b)

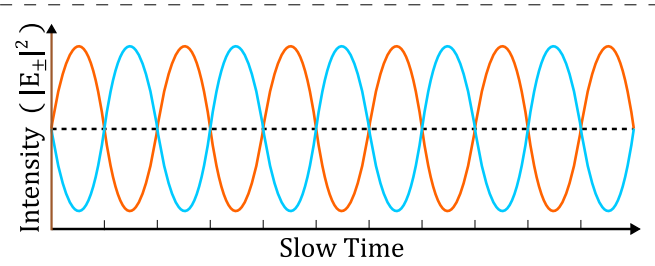

FIG. 1. (a) Schematic of a ring resonator. Two identical input beams enter the resonator, via a coupling mechanism, to then circulate in opposing directions. They each complete many round-trips before eventually leaving the resonator to continue to their respective outputs. The inset shows a glass microrod resonator (the bulge in the middle of the rod). (b) Example of antiphase periodic switching. "Slow time" refers to a timescale much larger than the round-trip time of the resonator. 
of temporal oscillations in their intensities [6]. The amplitudes of these oscillations grow and then switching of the dominant oscillating mode is observed. This "self-switching" of the dominant mode restores the symmetry between the time-averaged intensities, and is an example of attractor merging. Controllable switching between the two beams finds natural applications in the encoding of optical signals, chaotic cryptography, and waveform synthesizers. In addition to passive systems, our results may be of consequence for the gain dynamics of ring lasers [17,18], and for systems that host Kerr solitons [19-23]—especially those with counterpropagating modes [24]. We numerically model this self-switching behavior using a coupled dynamical system that features only the self- and cross-phase Kerr nonlinearities and provide an explanation for its occurrence. This demonstrates that, despite the inevitable presence of other nonlinear phenomena in the microresonator-including thermal nonlinearities, dispersion, and small amounts of frequency comb generation [25] the switching between the two counterpropagating beams can be ascribed to the Kerr effect. The model system comprises two coupled complex differential equations:

$$
\frac{d E_{ \pm}}{d t}=E_{\text {in }}-\left[1+i\left(\theta-A\left|E_{ \pm}\right|^{2}-B\left|E_{\mp}\right|^{2}\right)\right] E_{ \pm},
$$

where $E_{ \pm}$are the complex amplitudes of the clockwise and counterclockwise propagating fields seen in Fig. 1; $E_{\text {in }}$ is the envelope of the input beam(s); $\theta$ is the cavity detuning (the difference between the frequency of the input beam and the closest cavity resonant frequency); and finally, $A$ and $B$ are the self- and cross-phase modulation constants, respectively. The system is symmetric upon exchange of $E_{ \pm}$with $E_{\mp}$, and the simplest symmetric solutions are those where the two fields are equal and follow a common trajectory. Symmetry breaking in this system has recently been investigated in Refs. [6,26,27]. We consider $A=1$ and $B=2$, since our resonator is nondiffusive $[1,28]$. Equations (1) are mathematically equivalent to those describing two copropagating light components circulating within a Kerr ring resonator, with left and right circular polarizations, respectively $[6,26,29]$. Although we experimentally focus on the case of counterpropagating fields, similar effects to those described here should be observable in other systems, such as those in Ref. [30].

In Fig. 2, we plot the intensities of the two modes versus the detuning, $\theta$, for $E_{\text {in }}=2$. At the beginning, the two intensities are equal to each other (symmetric output). At $\theta=1.74$, spontaneous symmetry breaking occurs, leading to a dominant and a suppressed mode (solid lines in Fig. 2). This is followed by the onset of temporal oscillations for both modes at the outer edges of zones (1), and then by an intermediate region of partial overlap between the oscillating intensities of the two modes in zones (2).

Equations (1) have been shown to exhibit chaotic behavior after a rapid succession of period-doubling bifurcations $[6,26]$. It is, therefore, instructive to visualize this system with Poincare sections that sample the maxima and minima of the intensity oscillations-denoted by the red dots in Fig. 2. The dense bands of these dots suggest chaotic variation of the oscillating intensities. With a small change in detuning, these dense bands can give way to thin lines, implying the emergence of periodicity from a previously chaotic regime. By varying the detuning within chaotic oscillations, one enters (and leaves) a region of selfswitching between the two counterpropagating modessee zone (3) in green in Fig. 2. Here, self-switching between the modes can take forms in which the intensity variations are chaotic or periodic (many or few red dots,

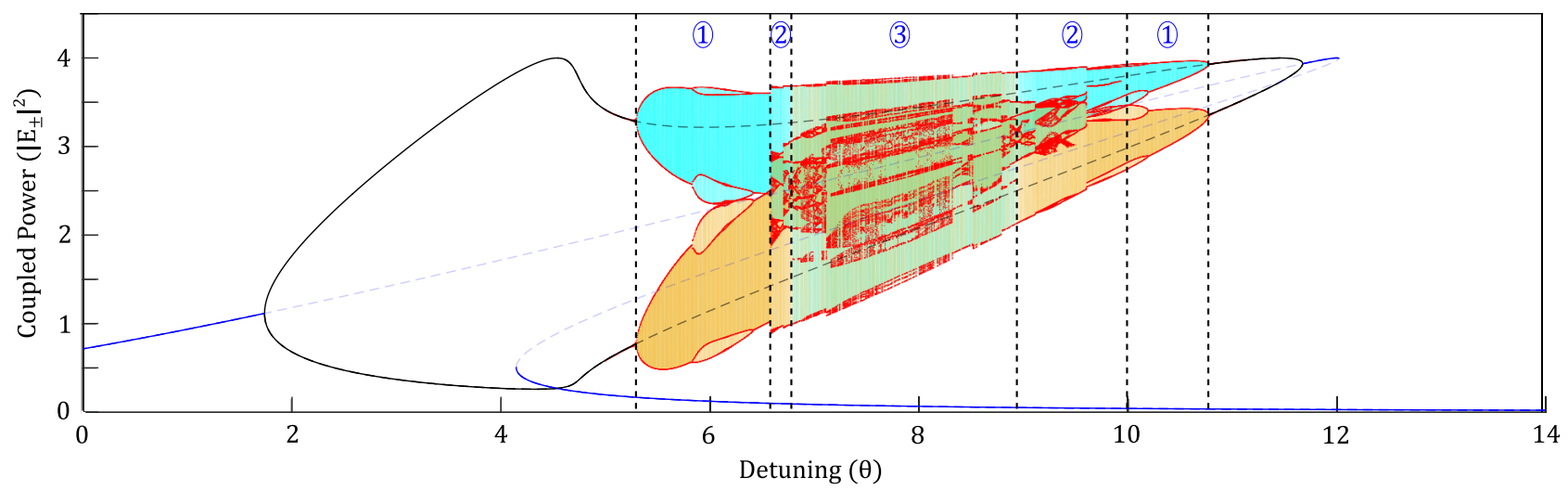

FIG. 2. Stationary and oscillatory intensities, described by Eqs. (1), under a forward detuning scan, with $\left|E_{\text {in }}\right|^{2}=4.0$. The blue and black lines indicate the symmetric and symmetry-broken stationary solutions, respectively, with solid lines indicating stable states and pale dashed lines indicating unstable states. The red dots denote Poincaré sections: a red dot is placed at each local minimum and maximum of an oscillation. Also shown, in semitransparent yellow and blue, are the full ranges of the oscillations. The yellow and blue shading results in a green colouring where the oscillations overlap. One observes three distinct regions: In zones (1), the oscillations in intensity do not overlap. In zones (2), they partially overlap, but this does not cause switching. In zone (3), switching occurs between the intensities of the two counterpropagating modes. 
respectively). Next, we shall investigate how this manifests in an experiment.

Our experimental setup uses a whispering-gallery mode microrod resonator $(1.9 \mathrm{~mm}$ in diameter), with a cavity half-linewidth, $\gamma$, of approximately $1 \mathrm{MHz}$. This was machined from a rod of fused silica, following the procedure in Ref. [31]. The resonator is pumped at $1.55 \mu \mathrm{m}$ with an amplified external-cavity diode laser, coupled via a tapered optical fiber. The optical circuitry of the setup is depicted in Fig. 3(a). The number of

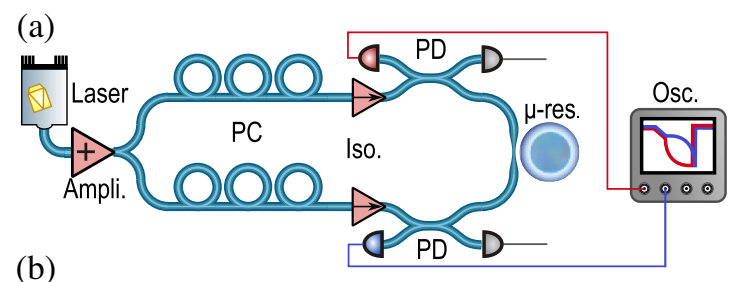

(b)
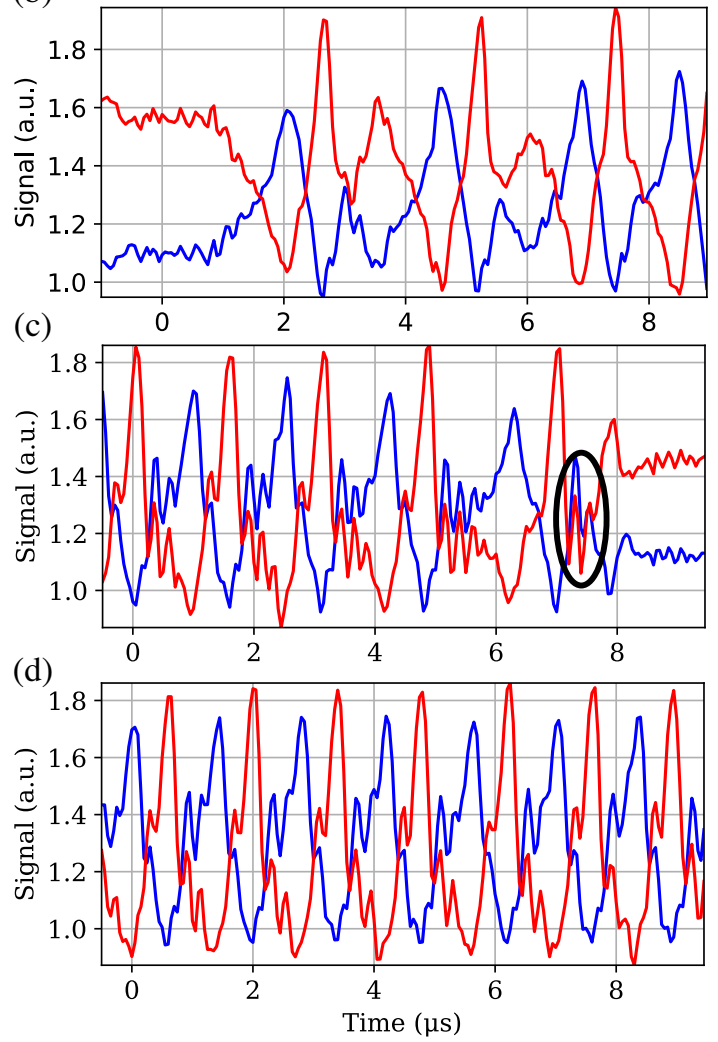

FIG. 3. (a) Schematic of the experimental setup. Fiber amplifier (Ampli.), polarization controllers (PC), optical isolators (Iso.), photodiodes (PD), microresonator ( $\mu$-res.), oscilloscope (Osc.). Power attenuation components are omitted for simplicity. (b)-(d) Examples of different experimentally observed selfswitching behaviors under a detuning scan of a single cavity resonance-the traces depict fluctuations in optical power. (b) Intermittent switching where the modes do not exchange dominance every time their intensities approach similar values. (c) Transient synchronization phenomena in between transition events (circled). (d) Near-periodic switching dynamics approach regular periodicity (the amplitudes of the intensity oscillations reach similar minima and maxima for every mode exchange). components is minimized in order to reduce optical losses, thereby maximising the power available to couple into the resonator, and so allowing easy access to symmetry-broken oscillatory and chaotic regimes. The power coupled into the resonator is inferred by subtracting the transmission signal in each direction from its respective baseline valuemeasured with the laser out of resonance. The input laser is scanned across the chosen cavity resonance. The polarization of each input branch is independently adjusted to maximize coupling efficiency and their relative powers are adjusted until the system begins spontaneously flipping between dominant directions of coupled light, indicating that they are sufficiently balanced to achieve spontaneous symmetry breaking. The oscillations within the symmetrybroken region are then investigated. Knowing that the input powers are balanced, the traces for each direction are subsequently rescaled to share a common zero and maximum coupled power, in order to correct for differences in the responses of the photodiodes. During scans of the input laser detuning, several regimes of self-switching oscillations are observed as displayed in Figs. 3(b)-3(d).

Intermittent switching.-By "intermittent," we refer to oscillations that do not exchange the dominant mode every time the intensities approach similar values. An example of this behavior is shown in Fig. 3(b), where the red trace remains dominant for two sequential peaks at a time.

Transient synchronization.-Another phenomenon that can occur is transient synchronization of the mode switching, followed temporarily by a loss of their antiphase relationship while spiraling towards the unstable symmetric solution, over a timescale of roughly the inverse of the halflinewidth, $1 / \gamma$. Since the symmetric state is unstable, the mode amplitudes subsequently fly apart. This is shown on the right-hand side of Fig. 3(c).

Near-periodic switching.-This is characterized by almost regular exchanges of the dominant mode, while the temporal evolutions of both modes remain chaotic [see Fig. 3(d)]. This regime is similar to, but distinct from, the one shown on the left in Fig. 3(c), as it shows minimal deviation from regular switching behavior. Ineliminable experimental noise may prevent a transition to fully periodic switching behavior.

Having observed different kinds of switching oscillations in the experimental microresonator, we use numerical integration of Eqs. (1) to reproduce their frequencies and structure, and to investigate their anticipated fully periodic switching behavior. Figures 4(a), 4(c), and 4(e) show examples of mode switching oscillations of the intensities while the corresponding rf intensity spectra are displayed in Figs. 4(b), 4(d), and 4(f). Intermittent switching in Figs. 4(a) and 4(b) results in broadband chaotic dynamics of the oscillations of the mode intensities. In Figs. 4(c) and 4(d), we observe near-periodic switching between the two intensities (see the lower part of the rf intensity spectrum). Adjustments of the detuning parameter can lead to fully 


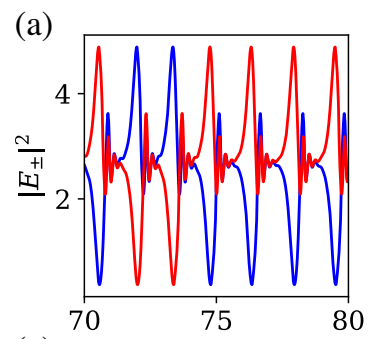

(c)

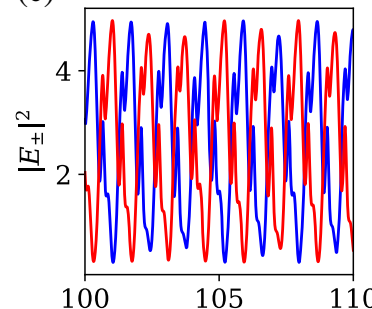

(e)

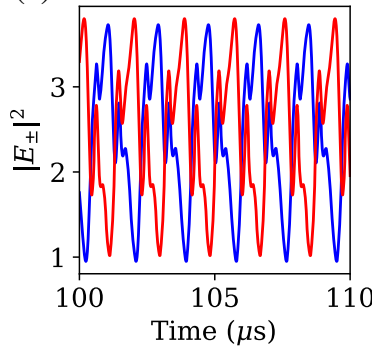

(b)

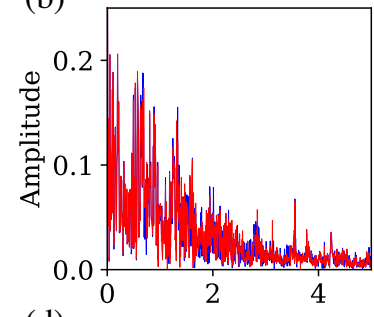

(d)

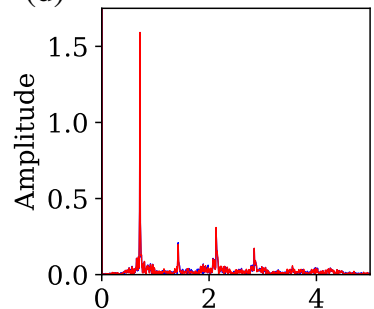

(f)

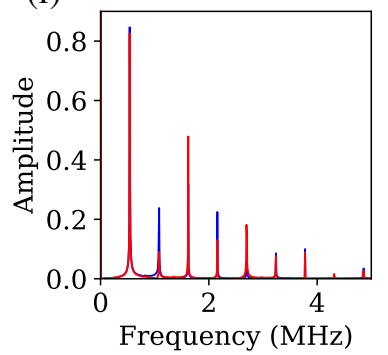

FIG. 4. Time series and rf intensity spectra (FFT of $\left|E_{ \pm}\right|^{2}$ ) of different switching states obtained by numerical integration of Eqs. (1). (a)-(b) Intermittent switching: the modes swap irregularly, with chaotic amplitude variations $\left(\left|E_{\text {in }}\right|^{2}=5.8, \theta=6.984\right)$. (c)-(d) Near-periodic switching approaches regular periodicity $\left(\left|E_{\text {in }}\right|^{2}=5.8, \theta=7.1\right)$. (e)-(f) Periodic switching (under imbalanced input powers), implying the disappearance of broadband chaos $\left(\left|E_{\mathrm{in}_{1}}\right|^{2}=4,\left|E_{\mathrm{in}_{2}}\right|^{2}=4.2, \theta=6.92\right)$.

periodic self-switching between the counterpropagating modes, even for imbalanced input powers [see Figs. 4(e) and 4(f)]. This total elimination of chaos is difficult to observe experimentally because of noise.

The full variety of different self-switching Kerr oscillations is presented in Fig. 5, where we compare and contrast the different dynamical regimes when changing the detuning, $\theta$, and the input power, $\left|E_{\text {in }}\right|^{2}$. In Fig. 5(a), we report their characteristic frequencies by extracting the dominant nonzero Fourier component from rf intensity spectra, such as those displayed in Figs. 4(b), 4(d), and 4(f). In the region of mode switching, the characteristic frequency lowers - see the purple area in Fig. 5(a) - to around half of the typical values before the onset of switching. In Fig. 5(b), where different dynamical behaviors are characterized by different colors, red regions of self-switching are clearly visible. Periodic switching, as displayed in Figs. 4(e) and 4(f), appears here sporadically.

The transition to self-switching behavior [see the boundaries of zone (3) in Fig. 2] implies that two symmetrybroken attractors-in which the time-averaged intensities
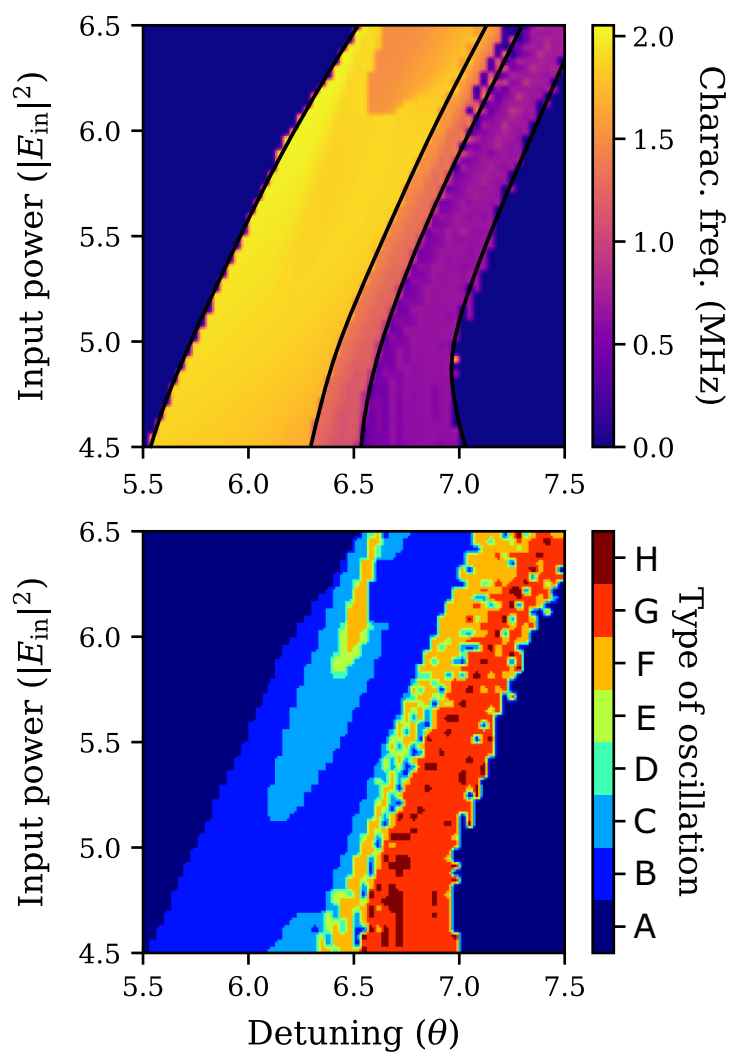

FIG. 5. Heat maps when varying the pump power and detuning. (a) Characteristic frequencies (key changes in behavior are marked with black curves). (b) Various types of dynamics: A, steady state; B, regular oscillations; C, period doubled; D, period tripled; E, period quadrupled; F, chaotic, nonswitching; G, chaotic switching; $\mathrm{H}$, periodic switching. The period-tripled state, like periodic switching, is associated with the total disappearance of chaos. The system reverts to steady-state behavior towards the right of the frames.

of the two modes are unequal [see zones (1) and (2) in Fig. 2] - merge into a single one where the symmetry in their intensities is restored on average. This is characteristic of a symmetry restoration crisis [32]. Within this selfswitching region, chaotic, intermittent, and periodic selfswitching take place on a single attractor that contains the trajectories of both $E_{ \pm}$and $E_{\mp}$. This implies that the originally broken symmetry is now restored on average over the period of the main component of oscillation.

We have experimentally observed self-switching behaviors in counterpropagating light-including near-periodic switching - using a passive microresonator with Kerr nonlinearity. We have also demonstrated that these behaviors can be explained by a simple dynamical system that considers only Kerr effects. In particular, the self-switching regime occurs in a well-defined region of the parameter space delimited by a symmetry restoring global bifurcation. This model also reproduces all the observed dynamical states of self-switching in the microrod resonatorincluding excellent agreement with the observed frequency 
of switching. It also predicts sporadic regimes of periodic switching that may be difficult to observe experimentally because of internal noise. Our results are of interest in the study of global bifurcations in dynamical systems, such as symmetry-restoring crises. From a practical perspective, self-switching periodicity of the counterpropagating modes can be applied in the controlled generation of twin waveforms and signal encoding, while chaotic states can be potentially employed in the generation of chaotic-cryptographic algorithms [33] as well as chaos-induced stochastic resonance [34].

All data underpinning this publication are openly available from the University of Strathclyde KnowledgeBase at [35].

We acknowledge financial support from: Engineering and Physical Sciences Research Council (EPSRC) DTA Grant No. EP/M506643/1; H2020 Marie Skłodowska-Curie Actions (MSCA) (748519, CoLiDR); National Physical Laboratory Strategic Research; H2020 European Research Council (ERC) (756966, CounterLight); EPSRC and the Scottish Universities Physics Alliance (SUPA). We would also like to thank Niall Moroney for his advice and assistance with some of the coding.

M. T. M. W. and L. H. contributed equally to this work.

*m.woodley20@imperial.ac.uk "pascal.delhaye@mpl.mpg.de

[1] A. Kaplan and P. Meystre, Opt. Commun. 40, 229 (1982).

[2] A. Kaplan and P. Meystre, Opt. Lett. 6, 590 (1981).

[3] L. Del Bino, J. M. Silver, M. T. M. Woodley, S. L. Stebbings, X. Zhao, and P. Del'Haye, Optica 5, 279 (2018).

[4] L. Del Bino, J. M. Silver, S. L. Stebbings, and P. Del'Haye, Sci. Rep. 7, 43142 (2017).

[5] E. M. Wright, P. Meystre, W. J. Firth, and A. E. Kaplan, Phys. Rev. A 32, 2857 (1985).

[6] M. T. M. Woodley, J. M. Silver, L. Hill, F. Copie, L. Del Bino, S. Zhang, G.-L. Oppo, and P. Del'Haye, Phys. Rev. A 98, 053863 (2018).

[7] K. Otsuka, Opt. Lett. 8, 471 (1983).

[8] W. Firth and C. Paré, Opt. Lett. 13, 1096 (1988).

[9] C. Wu, J. Fan, G. Chen, and S. Jia, Opt. Express 27, 28133 (2019).

[10] F. Copie, M. T. M. Woodley, L. Del Bino, J. M. Silver, S. Zhang, and P. Del'Haye, Phys. Rev. Lett. 122, 013905 (2019).

[11] A. E. Fomin, M. L. Gorodetsky, I. S. Grudinin, and V. S. Ilchenko, J. Opt. Soc. Am. B 22, 459 (2005).

[12] C. Bao, B. Shen, M.-G. Suh, H. Wang, K. Safak, A. Dai, A. B. Matsko, F. X. Kartner, and K. J. Vahala, Phys. Rev. A 103, L011501 (2021).
[13] G. Giacomelli, F. Marin, and I. Rabbiosi, Phys. Rev. Lett. 82, 675 (1999).

[14] M. Virte, K. Panajotov, H. Thienpont, and M. Sciamanna, Nat. Photonics 7, 60 (2013).

[15] J. Hillbrand, D. Auth, M. Piccardo, N. Opacak, E. Gornik, G. Stresser, F. Capasso, S. Breuer, and B. Schwarz, Phys. Rev. Lett. 124, 023901 (2020).

[16] A. Giraldo, B. Krauskopf, N. G. R. Broderick, J. A. Levenson, and A. M. Yacomotti, New J. Phys. 22, 043009 (2020).

[17] L. Gelens, S. Beri, G. V. der Sande, G. Mezosi, M. Sorel, J. Danckaert, and G. Verschaffelt, Phys. Rev. Lett. 102, 193904 (2009).

[18] A. Trita, G. Mezosi, M. J. Latorre-Vidal, M. Zanola, M. J. Strain, F. Bragheri, M. Sorel, and G. Giuliani, IEEE J. Quantum Electron. 49, 877 (2013).

[19] T. J. Kippenberg, A. L. Gaeta, M. Lipson, and M. L. Gorodetsky, Science 361, eaan8083 (2018).

[20] H. Bao, A. Cooper, M. Rowley, L. D. Lauro, J. S. T. Gongora, S. T. Chu, B. E. Little, G.-L. Oppo, R. Morandotti, D. J. Moss, B. Wetzel, M. Peccianti, and A. Pasquazi, Nat. Photonics 13, 384 (2019).

[21] H. Guo, M. Karpov, E. Lucas, A. Kordts, M. H. P. Pfeiffer, V. Brasch, G. Lihachev, V. E. Lobanov, M. L. Gorodetsky, and T. J. Kippenberg, Nat. Phys. 13, 94 (2017).

[22] J. K. Jang, A. Klenner, X. Ji, Y. Okawachi, M. Lipson, and A. L. Gaeta, Nat. Photonics 12, 688 (2018).

[23] G. Xu, A. Nielsen, B. Garbin, L. Hill, G.-L. Oppo, J. Fatome, S. G. Murdoch, S. Coen, and M. Erkintalo, arXiv:2008.13776.

[24] Q.-F. Yang, X. Yi, K. Y. Yang, and K. Vahala, Nat. Photonics 11, 560 (2017).

[25] P. Del'Haye, A. Schliesser, O. Arcizet, T. Wilken, R. Holzwarth, and T. J. Kippenberg, Nature (London) 450, 1214 (2007).

[26] L. Hill, G.-L. Oppo, M. T. M. Woodley, and P. Del'Haye, Phys. Rev. A 101, 013823 (2020).

[27] B. Garbin, J. Fatome, G.-L. Oppo, M. Erkintalo, S. G. Murdoch, and S. Coen, Phys. Rev. Research 2, 023244 (2020).

[28] W. Firth, I. Galbraith, and E. Wright, J. Opt. Soc. Am. B 2, 1005 (1985).

[29] J. Geddes, J. Moloney, E. Wright, and W. Firth, Opt. Commun. 111, 623 (1994).

[30] D. A. Mártin and M. Hoyuelos, Phys. Rev. A 82, 033841 (2010).

[31] P. Del'Haye, S. A. Diddams, and S. B. Papp, Appl. Phys. Lett. 102, 221119 (2013).

[32] A. Ben-Tal, Physica (Amsterdam) 171D, 236 (2002).

[33] J. Amigó, L. Kocarev, and J. Szczepanski, Phys. Lett. A 366, 211 (2007).

[34] F. Monifi, J. Zhang, S. K. Ozdemir, B. Peng, Y. Liu, F. Bo, F. Nori, and L. Yang, Nat. Photonics 10, 399 (2016).

[35] https://doi.org/10.15129/1d7eb435-719f-43a0-9709-3a33770 $6 \mathrm{ad} 82$. 